\title{
Association of Insulin Resistance with Overactive Bladder in Female Patients
}

\author{
Hakki Uzun, Adnan Yilmaz ${ }^{1}$, Ahu Kemik², Orhan Unal Zorba, Mehmet Kalkan ${ }^{3}$ \\ Departments of Urology and ${ }^{1}$ Biochemistry, Recep Tayyip Erdogan University School of Medicine, Rize; \\ ${ }^{2}$ Department of Biochemistry, Istanbul University Cerrahpasa Medical Faculty, Istanbul; \\ ${ }^{3}$ Department of Urology, Fatih University School of Medicine, Istanbul, Turkey
}

Purpose: Metabolic syndrome and obesity have been advocated to be risk factors for the development of overactive bladder (OAB). Additionally, insulin resistance is the underlying mechanism of metabolic syndrome. We aimed to investigate the association of insulin resistance with overactive bladder in female patients.

Methods: We prospectively conducted the study in our urology department. Female patients aged between 30 and 76 years old applied to our policlinics with or without $\mathrm{OAB}$ symptoms were enrolled. One hundred and twenty-two patients with $\mathrm{OAB}$ and 62 age-matched controls without $\mathrm{OAB}$ were included into the study. Fasting serum insulin, glucose, high-density lipoprotein (HDL-c), and triglycerides levels were measured. Insulin resistance value was obtained via the homeostasis model assessment of insulin resistance (HOMA-IR) calculator. The chi-square and Mann-Whitney $U$ tests were used to compare differences in variables.

Results: Serum insulin level was found higher in female patients with $\mathrm{OAB}(11.5 \pm 6.2 \mu \mathrm{U} / \mathrm{mL})$ relative to controls $(6.4 \pm 2.1 \mu \mathrm{U} /$ $\mathrm{mL}$ ), statistically significant $(\mathrm{P}=0.036)$. In addition, HOMA-IR was significantly found higher in the OAB group, 2.86 ( 0.76 to 17.04 ) in comparison to controls, 1.32 ( 0.67 to 224$), \mathrm{P}=0.018$. High-density lipoprotein cholesterol levels (HDL-c) were significantly found lower in females with $\mathrm{OAB}$.

Conclusions: Insulin resistance can be associated to overactive bladder and may play significant role in pathogenesis.

Keywords: Overactive urinary bladder; Insulin resistance; Metabolic syndrome

\section{INTRODUCTION}

Overactive bladder $(\mathrm{OAB})$, is a highly prevalent and disturbing disease with a profound impact on quality of life. Urinary urgency is the based symptom and usually accompanied by frequency and nocturia, with or without urgency urinary incontinence, in the absence of urinary tract infection or other obvious pathology [1]. The pathophysiology has been substantially poorly understood, and metabolic syndrome has been elucidated to play a significant role in etiopathogenesis [2].

Recent clinical studies have provided emerging evidence that metabolic syndrome and obesity may play a significant role in the development of lower urinary tract symptoms (LUTS) in men and $\mathrm{OAB}$ in females [2]. Metabolic syndrome is a cluster of visceral obesity, dyslipidemia, hypertension and glucose intolerance. Insulin resistance (IR) is the underlying pathogenesis and is likely to be a significant link between the components of the metabolic syndrome [3]. Sympathetic overactivity, proinflammatory status, oxidative stress and other pathological conditions are known to be associated to metabolic syndrome-IR state. In addition, these mechanisms are also supposed to be involved in the development of $\mathrm{OAB}$ [2].

We aimed to investigate the association of IR with $\mathrm{OAB}$ in female patients and clarify the underlying mechanisms that may
Corresponding author: Hakki Uzun

Department of Urology, Recep Tayyip Erdogan University School of Medicine, Rize Tip Fakültesi, Rize, Turkey

Tel: +90-532-374-8999 / Fax: +90-464-217-0370 / E-mail: hakuzun@yahoo.com Submitted: October 15, 2012 / Accepted after revision: December 5, 2012
This is an Open Access article distributed under the terms of the Creative Commons Attribution Non-Commercial License (http://creativecommons.org/licenses/by-nc/3.0/) which permits unrestricted non-commercial use, distribution, and reproduction in any medium, provided the original work is properly cited. 
link these two pathologic conditions.

\section{MATERIALS AND METHODS}

We prospectively conducted the study in our urology department. Female patients aged between 30 and 76 years old applied to our policlinics suffering from $\mathrm{OAB}$ symptoms or complaints other than LUTS were invited to participate in our study. $\mathrm{OAB}$ syndrome is defined according to International Continence Society 2010 definition as urinary urgency that is usually accompanied by frequency and nocturia, with or without urgency urinary incontinence, in the absence of urinary tract infection or other obvious pathology [1]. Patients with symptoms arising from childhood or any congenital, neurologic and severe psychiatric diseases were not enrolled. Subjects with urinary tract infection, or with diabetes mellitus, with pure stress or mixed urinary incontinence and with previous pelvic surgery were also excluded. In both groups we excluded the patients with visceral obesity below $85 \mathrm{~cm}$.

Female patients agreed to participate in the study had completed a 3 day frequency-volume chart and the quality of life measures including the $\mathrm{OAB}$ validated 8-question screener and patients with scores 8 or greater were acknowledged to have an $\mathrm{OAB}$. The control group was free of urgency or urgency incontinence. All patients in $\mathrm{OAB}$ group had urge urinary incontinence.

Demographic characteristics such as age, medical history, marital status, educational attainment, drugs on use, comorbidities, constipation and menopausal status were obtained. Pelvic examination was performed in each patient with LUTS. Body weights, heights and waist circumference were recorded and body mass index was calculated. Blood pressure of subjects without current antihypertensive drug therapy were also measured.

\section{Blood Collection}

Overnight fasting blood samples were collected from each subject with minimal stasis and serum was separated. Serum aliquots were then storaged at $-80^{\circ} \mathrm{C}$. Fasting serum insulin concentration was determined with the automated chemiluminescent immunoassay analyzer (The Architect i1000SR analyzer, Abbott Laboratories. Abbott Park, IL, USA). Serum concentrations $(\mathrm{mg} / \mathrm{dL})$ of glucose, high-density lipoprotein cholesterol, and triglycerides were measured by automated enzymatic photometry (Abbott Architect 16200, Abbott Diagnostics, Lake Forest, IL, USA). Homeostasis model assessment of insulin re- sistance (HOMA-IR) was used to quantify IR and values was obtained via the HOMA-IR calculator.

The study was approved by the Local Ethics Committee and informed consent was obtained from all patients.

\section{Statistical Analysis}

Chi-square and Mann-Whitney $U$ test was used for all of the analysis performed between patients with $\mathrm{OAB}$ and controls due to nonnormal distribution of the variables. Statistical calculations were performed using PASW ver. 18 (IBM Co., Armonk, NY, USA). The null hypothesis was rejected if P-value was $<0.05$.

\section{RESULTS}

One hundred and twenty-two patients with $\mathrm{OAB}$ in the study group and 62 age-matched controls without OAB were included into the study. Mean ages for the study group and controls were equivalent, $52.1 \pm 8.6$ years (range, 33 to 73 years) and $51.1 \pm 6.4$ years (range, 42 to 68 years), respectively. Mean serum insulin level was higher in female patients with $\mathrm{OAB}, 11.5 \pm 6.2$ (range, 3.3 to $50.2 \mu \mathrm{U} / \mathrm{mL}$ ) relative to controls, $6.4 \pm 2.1$ (range, 2.2 to $9.7 \mu \mathrm{U} / \mathrm{mL})$, statistically significant $(\mathrm{P}=0.036)$. In addition, HOMA-IR values were calculated and significantly found higher in $\mathrm{OAB}$ group, 2.86 (range, 0.76 to 17.04) in comparison to controls, 1.32 (range, 0.67 to 2.24 ), $\mathrm{P}=0.018$. Both groups were equivalent in terms of marriage status, smoking habitus, education level and constipation. Demographic and clinical characteristics of patients with and without OAB is shown in Table 1 .

Hypertension was found higher in the study group, but did

Table 1. Demographic and clinical characteristics of patients with and without overactive bladder (OAB)

\begin{tabular}{lccc}
\hline Variable & $\mathrm{OAB}(+)$ & $\mathrm{OAB}(-)$ & P-value \\
\hline No. & 122 & 62 & \\
Age $(\mathrm{yr})$ & $52.1 \pm 8.6$ & $51.1 \pm 6.4$ & 0.38 \\
Waist circumference $(\mathrm{cm})$ & $102.7 \pm 14.7$ & $98.1 \pm 11.6$ & 0.12 \\
Hypertension (\%) & 52.5 & 36.8 & 0.11 \\
Height $(\mathrm{cm})$ & $162 \pm 3.1$ & $164 \pm 3.2$ & 0.18 \\
Weight $(\mathrm{kg})$ & $83.2 \pm 10.4$ & $76.9 \pm 4.2$ & 0.16 \\
Body mass index $\left(\mathrm{kg} / \mathrm{m}^{2}\right)$ & $32.4 \pm 4.9$ & $29.2 \pm 3.1$ & 0.21 \\
Constipation $(\%)$ & 27 & 21 & 0.13 \\
Menopause $(\%)$ & 80 & 88 & 0.31 \\
\hline
\end{tabular}

Values are presented as mean \pm standard deviation unless otherwies indicated. 
Table 2. Laboratory findings of patients with and without overactive bladder $(\mathrm{OAB})$

\begin{tabular}{lccc}
\hline Variable & $\mathrm{OAB}(+)$ & $\mathrm{OAB}(-)$ & P-value \\
\hline Insulin $(\mu \mathrm{U} / \mathrm{mL})$ & $11.5 \pm 6.2$ & $6.4 \pm 2.1$ & 0.036 \\
HOMA- IR & $2.86(0.76-17.04)$ & $1.32(0.67-2.24)$ & 0.018 \\
Glucose $(\mathrm{mg} / \mathrm{dL})$ & $107.4 \pm 6.1$ & $94.5 \pm 7.3$ & 0.007 \\
HDL $(\mathrm{mg} / \mathrm{dL})$ & $49.3 \pm 12.3$ & $58.4 \pm 13.4$ & 0.015 \\
Total cholesterol $(\mathrm{mg} / \mathrm{dL})$ & $216.1 \pm 44.3$ & $218.5 \pm 28.3$ & 0.29 \\
Triglyceride $(\mathrm{mg} / \mathrm{dL})$ & $133.4 \pm 84.1$ & $124.3 \pm 45.3$ & 0.49 \\
LDL $(\mathrm{mg} / \mathrm{dL})$ & $138.5 \pm 33.4$ & $132.3 \pm 40.8$ & 0.78 \\
\hline
\end{tabular}

Values are presented as mean \pm standard deviation or mean (range). HOMA-IR, homeostasis model assessment of insulin resistance; HDL, high-density lipoprotein; LDL, low-density lipoprotein.

not reach statistical significance. Additionally, high-density lipoprotein levels were significantly found lower in females with OAB. Triglyceride and total cholesterol levels were statistically nonsignificant (Table 2). Both groups were equivalent in terms of visceral obesity and body mass index.

\section{DISCUSSION}

Initially, hyperinsulinaemia has been related as an etiologic risk factor for prostate growth [3]. Following this attractive study, many trials have revealed associations between visceral obesity or metabolic syndrome and LUTS [2]. In terms of relation to $\mathrm{OAB}$ visceral obesity, the core component of the metabolic syndrome, has been found to be a risk factor in both sexes, more pronounced for women, and finally metabolic syndrome has been strongly related to $\mathrm{OAB}$ in females [2]. Additionally, both IR and $O A B$ have an increasing prevelance with advancing age. On account of these cumulative evidence, IR as an underlying mechanism of metabolic syndrome can play a significant role in the etiopatogenesis of this prevalent disease. Likewise, in our small group HOMA-IR values were significantly found higher than controls. Visceral obesity, body mass index and hypertension were not found significant between the groups. However, these parameters were significantly found higher in our previous study [2]. IR is known highly correlated to visceral obesity and therefore, we excluded the patients with visceral obesity below $85 \mathrm{~cm}$ in this study.

Inflammation and oxidative stress has been advocated in the etiopathogenesis of OAB. In an epidemiologic survey Kupelian et al. [4] revealed an association between increasing C-reactive protein levels and $\mathrm{OAB}$ for both men and women and hypoth- esized a role of inflammation in the development of OAB. In addition, urine levels of inflammatory mediators were found elevated in female patients with OAB [5]. This association has been supported by animal studies. In fructose-fed rats, a common model used for metabolic syndrome, detrusor overactivity mainly by increased phasic contractions were demonstrated in 62.5 to $66.7 \%$ of the rats compared to none in controls [6,7]. Histological examination of the bladders revealed leukocyte infiltration around the endothelium of vessels and into the interstitial tissue, hypoxia and fibrosis [7]. Furthermore, increased muscarinic M2-3 receptor m-RNA and protein expression has been associated with evidence of detrusor overactivity and connected with metabolic syndrome [8]. In addition to relation of metabolic syndrome related chronic inflammation with $\mathrm{OAB}$, another IR related mechanism, oxidative stress, has also been advocated to play a significant role in the pathogenesis of OAB. It has been suggested that reactive oxygene species stimulate capsaicin-sensitive bladder afferent fibres and mediate detrusor overactivity in rats [8].

Obesity is no longer characterized as a chronic and systemic inflammatory disease where adipose tissue produces inflammatory mediators [9]. Adipose tissue is known to produce and secrete several inflammatory mediators and adipokines (cytokines) [9]. These mediators, such as interleukin 6 and tumor necrosis factor alfa and other adipokines induce oxidative stress and endothelial dysfunction [10]. Additionally, obesity give rise to increase in nonesterified fatty acids [10]. These free fatty acids and inflammatory mediators may activate serine kinases which phosphorylate serine residues of insulin receptor substrate-1 and cause IR [9]. Disrupt insulin signaling further aggravates oxidative stress and endothelial dysfunction [10]. It is known that chronic subclinical inflammation affects endothelial dysfunciton and is involved in aterosclerotic process. Furthermore, obesity associated free fatty acids and inflammatory mediators are also responsible for the development of components of metabolic syndrome, which are IR, dyslipidemia, and hypertension [10]. IR is the common link between obesity, the metabolic syndrome and profinlammatory state, free fatty acids, hypercoagulability, hypothalamic-hypophyseal adrenal axis and cellular oxidative stress [10].

It is known that IR cause sympathetic overactivity [11]. Additionally, Hubeaux et al. [12] reported that sympathetic overactivity is the main neuropathologic finding in patients with idiopathic OAB. Increased sympathetic activity of the bladder neck and prostate is also an independent etiologic factor for the 
development of LUTS in men [13]. Women with OAB have been reported to have hypoesthesia in the urethra and may have alterations in $\mathrm{C}$ fiber function which has been suggested to play a role in detrusor overactivity [14]. Furthermore, decreased perfusion of the bladder neck was observed in elderly females with LUTS [15]. Additionally, it is shown that chronic nitric oxide (NO) deficiency at trigon and urethra can trigger a persistent contraction leading to a detrusor overactivity [16]. Trigon and proximal urethra mediates the sympathetic transduction of the bladder and therefore can be the critical zone of the development of $\mathrm{OAB}$ in females. Additonally, benign prostatic hyperplasia is suggested to cause to detrusor overactivity and storage symptoms in men. This might be partly true, and metabolic syndrome related IR and chronic inflammation in bladder seperately might cause storage symptoms.

There is growing evidence that decreased bladder blood flow may contribute to detrusor overactivity in elderly men and women [15]. In addition, severity of LUTS has been found correlated with decreasing blood flow in bladder neck or urethra [15]. Animal studies have shown that moderate bladder ischaemia resulted in enhanced detrusor overactivity [17], muscarinic receptor overreactivity and smooth muscle instability [17, 18]. Additionally, IR is known to cause to endothelial dysfunction and play significant role in formation of atherosclerosis [19]. Nevertheless, chronic ischaemia might increasingly contribute to pathogenesis at the advanced or refractory form of the $\mathrm{OAB}$.

We suggest that ischaemic damage forms through the storage phase to the begining of micturition and the bladder reperfuses as the urine outflows. Reperfusion injury is known to cause to more severe damage in bladder than ischaemia alone [20] and results in production of oxidative radicals, activates $\mathrm{C}$ fiber afferents and induce detrusor overactivity and urinary frequency [8]. Men or women with OAB have frequent detrusor contractions and relaxations and higher intrabladder pressures compared to subjects with healthy daily micturitions, that means, more frequent daily ischaemic and reperfusion cycles. Additionally, ischaemia increases collagen deposition and impairs the fibroelasticity of the bladder wall and contributes to noncompliance [17]. Considerable evidence suggests a relationship between LUTS and erectile dysfunction, and chronic pelvic ischaemia can be the common pathophysiology of both disorders.

NO has been reported to modulate the storage phase of the micturition reflex [16]. Additionally, it was shown that long term NO deficiency sentitizes the bladder to muscarinic agonists and reduces b-adrenoreceptor mediated detrusor smooth muscle relaxations [16] and cause detrusor overactivity. These evidences point out that $\mathrm{OAB}$ may have close associations with chronic NO deficiency. IR is associated with a decrease in endothelial nitric oxide synthase phosphorylation and decreased endothelial NO production [21]. Additionally, oxidative stress reduces NO production [21]. NO pathway can be a significant intermediate pathophysiology of IR and chronic inflammationoxidative stress related development of $\mathrm{OAB}$.

$\mathrm{OAB}$ is suggested to be characterized with increase in bladder wall thickness and ultrasonographic bladder wall measurement is proposed to be used as a diagnostic tool. It is known that IR and oxidative stress reduce NO production [21]. NO has been shown to inhibit the bladder smooth muscle cell growth [16] and chronic NO deficiency may result in smooth muscle cell proliferation contributing to bladder wall thickness. Additionally, IR is associated with an increase in the level of insulinlike growth factor 1 (IGF-1) and IGF-1 m-RNA has been found increased in urinary bladder during hypertrophy of smooth muscle and has been suggested to play a role in smooth muscle cell hypertrophy [22]. Furthermore, streptozotocin induced diabetic rats showed an increase in total bladder tissue with hypertrophy of the bladder wall and increase in smooth muscle and urothelium thickness [23]. All these IR related mechanisms might be the underlying pathophysiology of bladder wall thickness in patients with OAB. Moreover, it is shown that metabolic syndrome rabbits developed fibrosis in bladder [24], and ischaemia is shown to increase collagen content and impair the fibroelastic structure of the bladder [17] and results in thickening. Increase in collagen content decreases the viscoelasticity of the bladder wall and may result in low bladder compliance. Sudden increase in bladder pressure disproportional to bladder volume is the clinical definition of $\mathrm{OAB}$ and we believe corresponds to a hypocompliance. IR and chronic inflammation can decrease the compliance of the bladder through impairing the viscoelastic structure of the bladder wall.

Based on these evidences, $\mathrm{OAB}$ might be the pathology of all layers of bladder wall rather than the sole smooth muscle (myogenic theory) or nerves (neurogenic theory), and $\mathrm{OAB}$ can be an impaired bladder contractility rather than an overactivity.

IR is found to stimulate ovarian androgenesis [25] and significant positive correlation was found between total testosterone level and urgency and nocturia in females with a IR related disease, polycystic ovary syndrome, and indicates that women 
with higher serum testosterone levels are more likely to have bladder symptoms [26]. In contrast, the result of IR in men is a relative hyperestrogenemia which is reported to be related to detrusor overactivity in rats [27].

Urinary nerve growth factor (NGF) levels are reported to be increased in women with $\mathrm{OAB}$ [28] and serum levels were upregulated in metabolic syndrome [29]. NGF is mainly implicated in inflammatory response and NGF gene and protein is reported to be upregulated in ischaemic bladder [18] and elevated urinary levels can be the result of obesity-IR state.

The limitations of our study are the nature of cross-sectional and small size of the patient cohort, and lack of measurement of inflammatory mediators. Further studies are needed to investigate the pathogenetic relations of IR and chronic inflammation with $\mathrm{OAB}$.

People with nocturia have been shown to have higher mortality rates [30] and IR can be the underlying etiologic reason. IR can also be cause of a likely higher mortalilty in women with OAB. Cardiovascular diseases, erectile dysfunction, diabetes, and Alzheimer's disease are related to IR and we believe idiopathic OAB can be a predictor of general health status. Additionally, improved control of IR, obesity related chronic inflammation and oxidative stress might prevent the onset of $\mathrm{OAB}$ or improve the symptoms.

In conclusion, IR is associated to OAB and may play significant role in pathogenesis.

\section{CONFLICT OF INTEREST}

No potential conflict of interest relevant to this article was reported.

\section{REFERENCES}

1. Haylen BT, de Ridder D, Freeman RM, Swift SE, Berghmans B, Lee J, et al. An International Urogynecological Association (IUGA)/International Continence Society (ICS) joint report on the terminology for female pelvic floor dysfunction. Neurourol Urodyn 2010;29: 4-20.

2. Uzun $\mathrm{H}$, Zorba OU. Metabolic syndrome in female patients with overactive bladder. Urology 2012;79:72-5.

3. Hammarsten J, Hogstedt B. Hyperinsulinaemia as a risk factor for developing benign prostatic hyperplasia. Eur Urol 2001;39:151-8.

4. Kupelian V, Rosen RC, Roehrborn CG, Tyagi P, Chancellor MB, McKinlay JB. Association of overactive bladder and C-reactive pro- tein levels. Results from the Boston Area Community Health (BACH) Survey. BJU Int. 2012;110:401-7.

5. Tyagi P, Barclay D, Zamora R, Yoshimura N, Peters K, Vodovotz Y, et al. Urine cytokines suggest an inflammatory response in the overactive bladder: a pilot study. Int Urol Nephrol 2010;42:629-35.

6. Tong YC, Cheng JT. Alterations of M2,3-muscarinic receptor protein and mRNA expression in the bladder of the fructose fed obese rat. J Urol 2007;178(4 Pt 1):1537-42.

7. Lee WC, Chien CT, Yu HJ, Lee SW. Bladder dysfunction in rats with metabolic syndrome induced by long-term fructose feeding. J Urol 2008;179:2470-6.

8. Masuda H, Kihara K, Saito K, Matsuoka Y, Yoshida S, Chancellor $\mathrm{MB}$, et al. Reactive oxygen species mediate detrusor overactivity via sensitization of afferent pathway in the bladder of anaesthetized rats. BJU Int 2008;101:775-80.

9. Bastard JP, Maachi M, Lagathu C, Kim MJ, Caron M, Vidal H, et al. Recent advances in the relationship between obesity, inflammation, and insulin resistance. Eur Cytokine Netw 2006;17:4-12.

10. Gallagher EJ, Leroith D, Karnieli E. The metabolic syndrome: from insulin resistance to obesity and diabetes. Med Clin North Am 2011; 95:855-73.

11. Landsberg L. Role of the sympathetic adrenal system in the pathogenesis of the insulin resistance syndrome. Ann N Y Acad Sci 1999; 892:84-90.

12. Hubeaux K, Deffieux X, Raibaut P, Le Breton F, Jousse M, Amarenco G. Evidence for autonomic nervous system dysfunction in females with idiopathic overactive bladder syndrome. Neurourol Urodyn 2011;30:1467-72.

13. McVary KT, Rademaker A, Lloyd GL, Gann P. Autonomic nervous system overactivity in men with lower urinary tract symptoms secondary to benign prostatic hyperplasia. J Urol 2005;174(4 Pt 1):1327433.

14. Kenton K, Lowenstein L, Simmons J, Brubaker L. Aging and overactive bladder may be associated with loss of urethral sensation in women. Neurourol Urodyn 2007;26:981-4.

15. Pinggera GM, Mitterberger M, Steiner E, Pallwein L, Frauscher F, Aigner F, et al. Association of lower urinary tract symptoms and chronic ischaemia of the lower urinary tract in elderly women and men: assessment using colour Doppler ultrasonography. BJU Int 2008;102:470-4.

16. Monica FZ, Bricola AA, Bau FR, Freitas LL, Teixeira SA, Muscara $\mathrm{MN}$, et al. Long-term nitric oxide deficiency causes muscarinic supersensitivity and reduces beta(3)-adrenoceptor-mediated relaxation, causing rat detrusor overactivity. Br J Pharmacol 2008;153: 1659-68. 
17. Azadzoi KM, Tarcan T, Kozlowski R, Krane RJ, Siroky MB. Overactivity and structural changes in the chronically ischemic bladder. J Urol 1999;162:1768-78.

18. Azadzoi KM, Chen BG, Radisavljevic ZM, Siroky MB. Molecular reactions and ultrastructural damage in the chronically ischemic bladder. J Urol 2011;186:2115-22.

19. Baron AD. Insulin resistance and vascular function. J Diabetes Complications 2002;16:92-102.

20. Bratslavsky G, Kogan BA, Matsumoto S, Aslan AR, Levin RM. Reperfusion injury of the rat bladder is worse than ischemia. J Urol 2003;170:2086-90.

21. Duckles SP, Miller VM. Hormonal modulation of endothelial NO production. Pflugers Arch 2010;459:841-51.

22. Chen Y, Bornfeldt KE, Arner A, Jennische E, Malmqvist U, Uvelius $B$, et al. Increase in insulin-like growth factor I in hypertrophying smooth muscle. Am J Physiol 1994;266(2 Pt 1):E224-9.

23. Pitre DA, Ma T, Wallace LJ, Bauer JA. Time-dependent urinary bladder remodeling in the streptozotocin-induced diabetic rat model. Acta Diabetol 2002;39:23-7.

24. Morelli A, Comeglio P, Filippi S, Sarchielli E, Cellai I, Vignozzi L, et al. Testosterone and farnesoid X receptor agonist INT-747 counteract high fat diet-induced bladder alterations in a rabbit model of metabolic syndrome. J Steroid Biochem Mol Biol 2012;132:80-92.

25. Mauras N, Welch S, Rini A, Haymond MW. Ovarian hyperandrogenism is associated with insulin resistance to both peripheral carbohydrate and whole-body protein metabolism in postpubertal young females: a metabolic study. J Clin Endocrinol Metab 1998; 83:1900-5.

26. Sahinkanat T, Ozturk E, Ozkan Y, Coskun A, Ekerbicer H. The relationship between serum testosterone levels and bladder storage symptoms in a female population with polycystic ovary syndrome. Arch Gynecol Obstet 2011;284:879-84.

27. Chavalmane AK, Comeglio P, Morelli A, Filippi S, Fibbi B, Vignozzi $\mathrm{L}$, et al. Sex steroid receptors in male human bladder: expression and biological function. J Sex Med 2010;7:2698-713.

28. Liu HT, Chen CY, Kuo HC. Urinary nerve growth factor in women with overactive bladder syndrome. BJU Int 2011;107:799-803.

29. Bullo M, Peeraully MR, Trayhurn P, Folch J, Salas-Salvado J. Circulating nerve growth factor levels in relation to obesity and the metabolic syndrome in women. Eur J Endocrinol 2007;157:303-10.

30. Kupelian V, Fitzgerald MP, Kaplan SA, Norgaard JP, Chiu GR, Rosen RC. Association of nocturia and mortality: results from the Third National Health and Nutrition Examination Survey. J Urol 2011; 185:571-7. 